\title{
BlueFriend: Using Bluetooth Technology for Mobile Social Networking
}

\author{
Patricia Tamarit, Carlos T. Calafate, Juan-Carlos Cano, Pietro Manzoni \\ Department of Computer Engineering \\ Technical University of Valencia, Spain \\ E-mail: patricia.tamarit@gmail.com, \{calafate, jucano, pmanzoni\}@disca.upv.es
}

\begin{abstract}
In this paper we present BlueFriend, a novel application for mobile devices that takes advantage of Bluetooth functionalities to create mobile social networks. Our application runs on PDAs and smart phones equipped with a Bluetooth adapter. BlueFriend periodically scans the environment in search for other members of the BlueFriend community; when found, "virtual personal cards" (VPCs) are exchanged with information about the user profile, the user preferences, likes and dislikes, etc. to assess the degree of matching among nearby users. High degrees of matching result in the exchange of personal information and contact details so that connected users are aware of how to reach each other in the future. Since Bluefriend relies on a widely available technology such as Bluetooth, its use can be easily extended.

BlueFriend combines the efforts to promote human relationships in virtual reality environments, such as Second Life's virtual community, while targeting real-life scenarios, thus embracing Weiser's vision of an ubiquitous computing world.
\end{abstract}

Keywords: mobile social networks, pervasively generated content, Bluetooth.

\section{Introduction}

Social networks are growing day-by-day, having a broad impact on our societies. In particular, mobile social networks offer a novel user-interaction paradigm that combines the benefits of social networks (e.g., Facebook [1] and Twitter [2]) with mobile computing devices.

Devices such as smart phones, PDAs and portable tablet PCs have become everyday-tools for many people. The ever increasing capabilities of these devices allow to extend their use to many novel applications in areas such as healthcare, entertainment, e-transactions, etc. Mobile social software [3] refers to software designed to explore the social possibilities of urban environments through mobile devices. One of the basic requirements of such software is that it must be very simple to use and efficient to operate, so that it integrates seamlessly into users' lives.

The low cost and low battery consumption of Bluetooth devices, allied with novel functionalities such has device and service discovery, has promoted the widespread adoption of this technology, allowing developers to design novel mobile applications that apply novel networking concepts to the real world. Nowadays most computing devices with networking requirements are equipped with a Bluetooth interface. In particular, the integration of this technology on mobile phones has increased the number of potential users to millions in a very short period of time.

In this paper we present BlueFriend, a Bluetooth-based mobile application designed to promote human relationships based on the matching of user profiles in the same social community. Contrarily to other types of applications (e.g., [4]) that promote a sedentary conduct and intensive use of the mobile terminal, BlueFriend follows just the opposite philosophy by promoting nomadism and requiring minimal user intervention on the mobile terminal. In fact, the chances of finding affine users within the community are increased as the user moves around in crowded areas since the user profile matching process only takes place when two community members are within Bluetooth's transmission range (usually around 10 meters) [5]. So, in places such as subways, stadiums, malls and entertainment areas, the activity of BlueFriend is expected to be high, while at home it is expected to be null.

The user profile matching process computes a matching index through a weighted averaging of individual comparisons for the different profile areas; the latter include preferences related to hobbies, music, sports, etc. If this matching index surpasses a certain user-defined threshold, the application proceeds to exchange personal and contact data to make it possible for both users to meet afterwards.

This paper is organized as follows: in the next section we refer to other related works in this field. Our proposal is presented in section 3, including some implementation details and screenshots. Section 4 addresses privacy, an important 
requirement for this sort of applications. In section 5 we make a performance assessment of our implementation using different PDA models. Finally, section 6 concludes the paper.

\section{Related work}

Applications that try to create mobile social networks have been around for some years, and new ones are evolving rapidly. Examples of such applications are Nokia Sensor [6], Mobiluck [7], Brightkite [8] and Loopt [9]. Whereas the aforementioned applications provide an opportunity for getting in touch with nearby people, they do not automate the process of finding users with similar interests through profile comparisons.

In [10], Paulos and Goodman explore the often ignored yet real relationships with the so called "Familiar Strangers". They describe several experiments and studies that led to designs for both a personal, body-worn, wireless device and a mobile phone based application that extend the Familiar Stranger relationship while respecting the delicate, yet important, constraints of our feelings and affinities with strangers in public places. However, authors do not propose any sort of interest matching technique among the elements of the social network.

Telelogs [11] is a system designed to serve as a medium through which individuals in urban spaces can communicate their thoughts and ideas with others within their environment, resulting in a better sense of community solidarity. It has taken the essence of the Blog, and transferred it to an audio format and made it accessible through one of the most ubiquitous mobile devices in the urban setting, the cell phone. Again, this proposal does not focus on binding users according to their interests either.

Serendipity [12] is an application that combines the existing communications infrastructure with online introduction systems' functionality to facilitate interactions between physically proximate people through a centralized server. The application repeatedly scans for Bluetooth devices, and transmits the discovered BTIDs to a server. Serendipity receives the BTID and threshold variables from the phones and queries a MySQL database for the user profiles associated with the discovered BTID addresses. If a profile exists, the system calls another script to calculate a similarity score between the two proximate users. When this score is above both users' thresholds, the script sends an alert to their phones with the other user's picture, their commonalities, a list of talking points, and additional contact information (at each user's discretion). The main drawbacks of this proposal are that a server is required to perform the matching of user interests, and that a communications infrastructure must be available to transmit the data collected.

Bluedating [13] is an alternative to Serendipity where users enter their interests and desires, as well as a profile of their desired partner. The system advertises this information (and only this information) over Bluetooth. A second part of the application continually searches for other profiles over Bluetooth. When it finds one, it compares the discovered profile to the desired profile. If the two profiles match, the system informs both users (usually by vibrating the phone) of the potential match. The main drawback of this solution is that is does not embrace Weiser's vision of a calm technology, potentially requesting user intervention frequently, especially in crowded places. Also, the proposal does not include a validation of their solution to assess whether it is able to perform adequately in environments where the number of users is high.

\section{BlueFriend}

BlueFriend is an application that takes advantage of mobile devices and Bluetooth technology to promote social networks, thus allowing to generate what is usually referred to as a mobile social network. The main goals sought are: (i) the application must be able to match user interests locally, (ii) no communications infrastructure should be required, thus allowing the application to operate in places where it is not available, or when the costs involved are too high, (iii) no user intervention shall be required at any moment during its execution, and (iv) the implementation must be efficient for it to be able to scale to large numbers of users, and also adopt multi-threading to handle parallel profile matching.

With BlueFriend all the contents exchanged are generated by the user. One of the important properties of this application is that its operation relies heavily on geographic context. In fact, user profiles are only exchanged when two members of BlueFriend's social community are nearby (within Bluetooth's radio range, which is about 10 meters). Another important property has to do with its mode of operation; contrarily to most social networks, where the user himself has to interact intensively with the network to participate actively [14], with BlueFriend all the tasks are performed automatically by the mobile terminal. So, after the user introduces his personal data and profile, the application will move into the background, operating by continuously trying to detect other members of the community and exchanging profiles.

A prototype of the BlueFriend application was developed using the .NET platform for devices running the Windows Mobile operating system (Pocket PC 2003 or superior) ${ }^{1}$. The graphical user interface (GUI) supports three different languages: English, Spanish, and Valencian (a local lan-

\footnotetext{
${ }^{1}$ BlueFriend can be downloaded for free at http://www.grc.upv.es/software/
} 
guage), and it can be easily extended to incorporate more languages.

In terms of requirements, the mobile terminal just has to include a Bluetooth interface for communication with other users. There is no special requirement for storage or memory since they have been reduced to a minimum to accommodate to the limitations of mobile terminals.

When users start the application they are presented with a user/password request for credentials. Afterwards they reach the main menu, where they can access all the relevant data. The application's functionality can be split into three main parts: user characterization, profile exchange/matching and result visualization.

\subsection{User characterization}

BlueFriend's framework allows the user to provide a detailed characterization about himself. This is achieved by filling-in different forms that allow generating a personal data card. The information gained by the application can be split into two different categories:

- Personal Data: where the user will insert private data about himself; except for age and gender, these data will not be sent to other community members unless there is a match.

- Profile: in this category the user will introduce public data about himself, such as his/her tastes and preferences in different areas. The profile is exchanged with all users to compute the matching index and it contains no personal details.

Figure 1 illustrates the user characterization process by representing a subset of the different menus available.

Personal data details are split into three main parts: contact details, user description and photo. The first part includes name, alias, gender, age, date of birth and contact e-mail. The user then proceeds to describe himself by introducing height, look, color and hair length, eye color, style, physical appearance, personality and whether he is a smoker or not. Finally the user has the possibility of including a photo.

The second part of user characterization, the profile, is fundamental since it allows the application to obtain reliable and detailed results during the user matching process. In this part the user specifies his interest with respect to six areas: music, movies, animals, hobbies (see figure 1, right), night life and sports. The user must simply state what his real interests are to find community members with similar characteristics. This is done by selecting, for each area, all the items of interest from a pre-defined list made available by the application. Notice that, despite the profile characterization could easily be extended to support other areas,

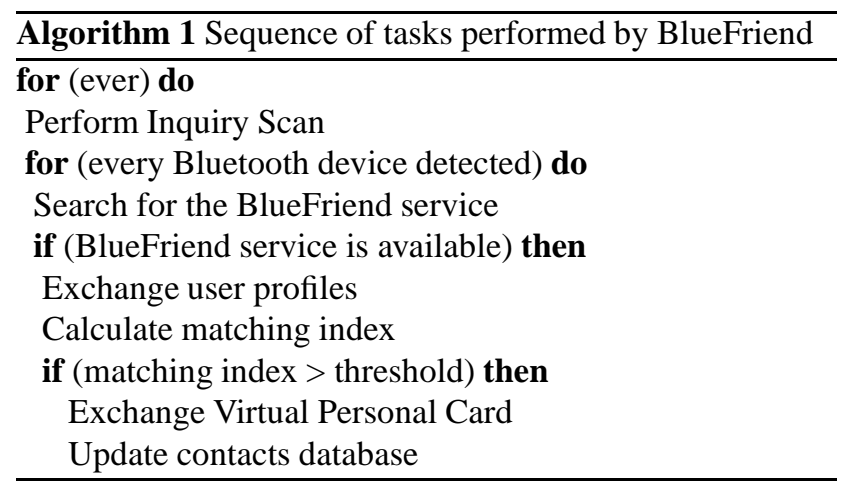

we believe that the ones proposed suffice for our proof-ofconcept development.

We use the XML language to store and exchange user preferences, so that extensions to the application can be made quite straightforward.

\subsection{Profile exchange and matching}

Once users fill-in their personal data cards for the first time, that information is stored on the mobile device and used to compute the matching index. Additionally, the user can refine the search process by restricting it according to objective criteria, such as gender or age range, and finally setting the minimum threshold for the ranking index in order to allow contact details to be exchanged.

From that point on, BlueFriend's search activity can be started. The sequence of tasks is described in algorithm 1. Basically, the search process will try to find nearby devices continuously. Only when it finds a device with the BlueFriend Service active does it exchange the user's profile (see figure 2).

Afterwards, a data analysis procedure starts to calculate the matching rank. One of our goals was to keep this analysis as short as possible to be effective while users are moving. So, the data analysis starts by checking whether the nearby users are within the desired search scope in terms of age and gender. Only if a user passes this filter does it proceed to calculate the matching index. A sub-index $S_{n}$ is calculated for each profile category in order to obtain partial values of matching rank. This is done according to the following formula:

$$
S_{n}=\frac{\left\{P_{n}^{A}\right\} \bigcap\left\{P_{n}^{B}\right\}}{\left\{P_{n}^{A}\right\} \bigcup\left\{P_{n}^{B}\right\}},
$$

where $\left\{P_{n}^{i}\right\}$ corresponds to the pool of elements chosen by user $i$ within category $n$. To compute the final matching index, the sub-indexes for each profile category $\left(S_{n}\right)$ are weighted so as to reward the similarities between users.

In case the matching index is above the threshold value 

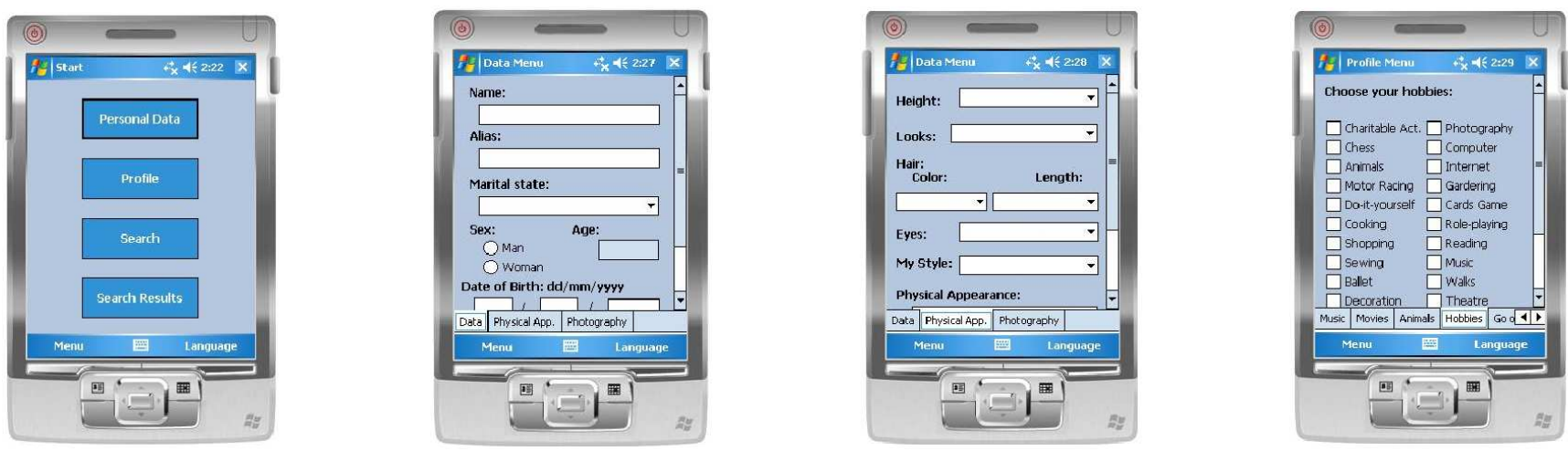

Figure 1. Setting the user's personal data and profile with BlueFriend.

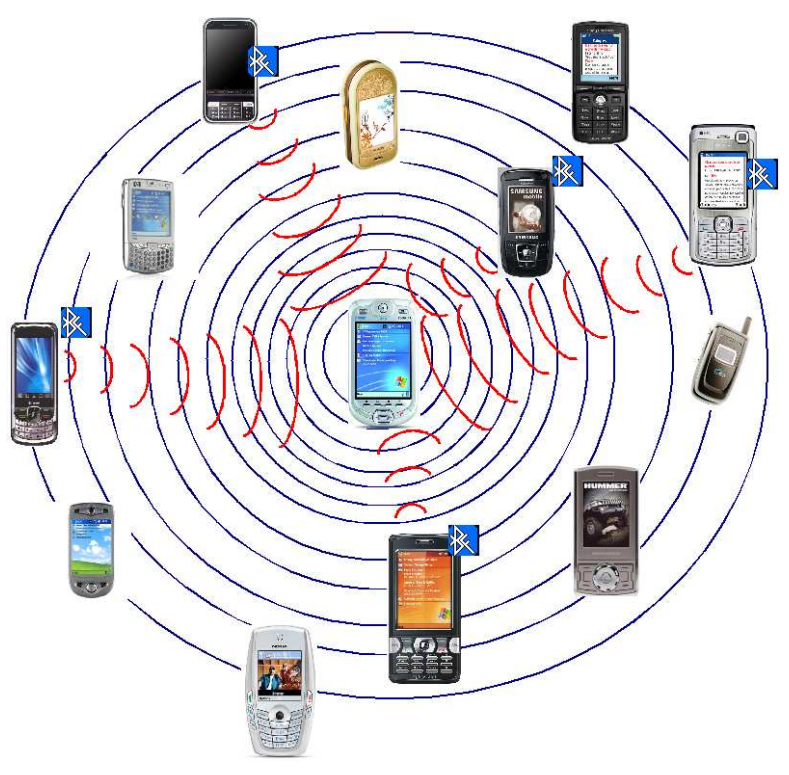

Figure 2. Environment scan for BlueFriendenabled mobile devices. defined by both users (meaning that the highest value prevails), another data exchange takes place, this time to include contact data. This second phase assures that private data is only exchanged among users when it is of common interest for both parties.

Our experiments have showed that, in the case of a positive match between two community members, the entire process including data exchanges and profile matching takes just a few seconds. Such low latency allows the application to scale-up in environments where the potential number of users is very high, as desired.

\subsection{Data gathering and visualization}

Bluefriends' data gathering process is completely automatic, not requiring any user intervention at all. Through a very intuitive user interface the user has full control of the application, being able to check its status and stopping its activity at any time.

Through the result visualization panel, the application reports to the user all the successful matches found in the database. Each entry contains a Virtual Personal Card with personal information and contact details, along with the matching index and all the partial values of the matching rank with respect to that particular member of the BlueFriend community .

Figure 3 illustrates what two different users get after a successful matching process with each other through BlueFriend. The application highlights the photo, name and matching index for that person. A detailed description of their main characteristics follows, and finally detailed percategory matching indexes are available.

Afterward the user can proceed to establish contact directly (e.g., through a phone call or an e-mail) or indirectly through, e.g., an invitation to a social network such as Facebook. 


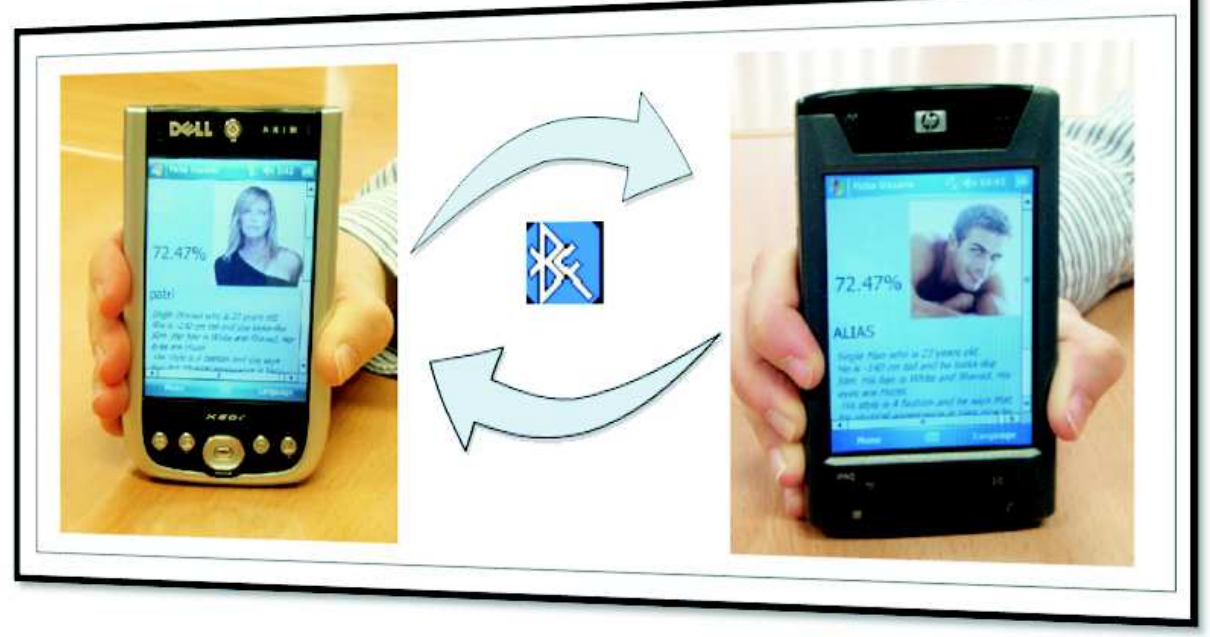

Figure 3. Result visualization in terms of personal data and profile after a successful matching between two members of the BlueFriend community.

\section{A note on users' privacy}

The anonymity provided by social networks represents a captivating factor for many sectors of our societies. As occurs with all novel technologies, users do not always take advantage of it in noble ways. In fact, there is a growing concern about providing private details to unknown users, since these could provide hints or clues for criminal activities. A possible solution to this problem could be relying on a centralized certification authority that made it possible to generate and exchange personal cards securely and confidently.

Compared to traditional social networks, the use of mobile social networks entails further problems since mobile terminals are more prone to being lost or robbed. In those cases, assailants could easily take advantage of their victim by having direct access to private data, including both personal information and contacts. Relying on strong encryption codes or storing critical information on external servers alone are possible solutions to this problem.

As a final remark, we consider that, independently of the technical solution offered, building-up people's trust on these new technologies through embedded security policies is vital for them to enjoy a wide acceptation from users worldwide.

\section{Performance assessment}

To further validate our proposed application we set forth a series of experiments whose primary goal was assessing the global time overhead, as well as the overhead associated to different tasks. For our tests we disposed of two PDA models: Dell Aximx50v and Qtek 200; these models have quite heterogeneous characteristics in terms of hardware, which aided at observing typical ranges of values. In figure 4 we show the average time involved with each task on each PDA model and mean values on the right. All values represented are the mean over 100 independent tests. Label "BF Service" refers to the time spent while searching for the Bluefriend service, and represents about $27 \%$ of the time. Labels " 2 WR" and " 2 RD" refer to the time spend during the profile exchange process, which requires two read and two write operations when a match is found; these represent $22 \%$ and $25 \%$ of the total time, respectively. Finally, the matching process ("MATCH") and the storage of the results ("STORAGE") represent the lowest time shares, taking $17 \%$ and $8 \%$ of the total time, respectively.

Despite results of figure 4 are interesting to better grasp the impact of the steps involved, in terms of application deployment it is also important to analyze the performance when we have more than one member of BlueFriend's community in our vicinity. In figure 5 we show the results when varying the total number of nearby users between 2 and 4 , also testing different matching possibilities.

We can observe that, thanks to the multi-threaded nature of the application, increasing the number of terminals does not incur into a proportional overhead increase, being all values within a quite reasonable time range.

We are currently using Bluefriend in Computer Networks classes at the Faculty of Informatics of our institu- 


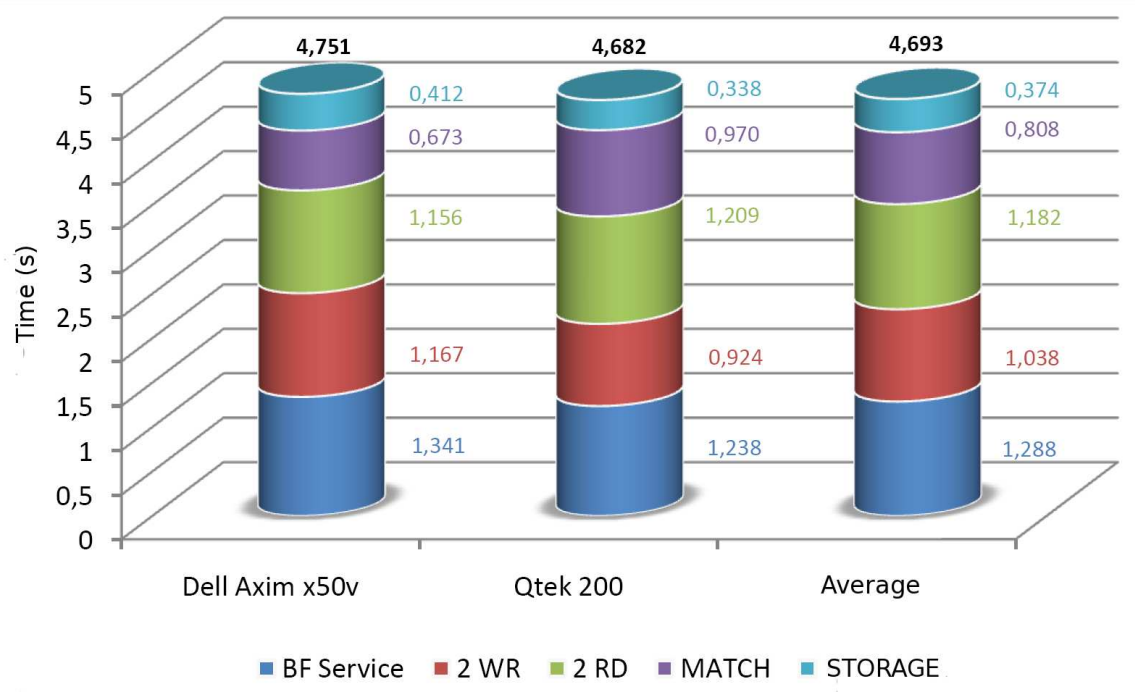

Figure 4. Cumulative bar chart describing the time overhead associated with the different tasks involved.

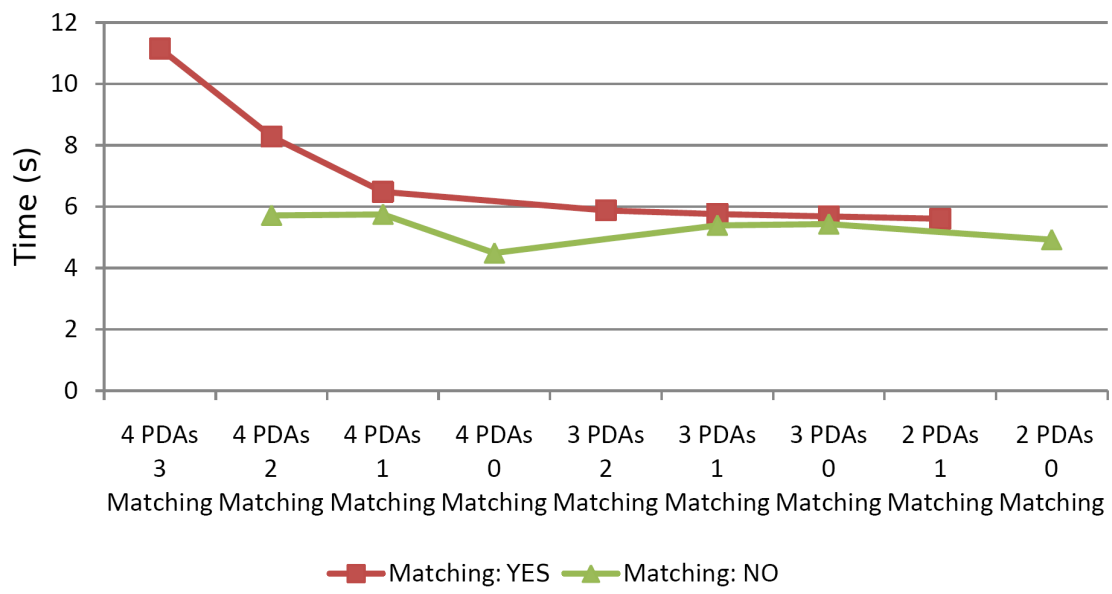

Figure 5. Total time overhead involved under different environment conditions. 
tion (Technical University of Valencia) as an example of how to apply novel networking concepts to different realworld needs. Our students have been asked to install and validate this application; their reports on how intuitive and functional it is have encouraged us to provide further developments in this research area.

\section{Conclusions}

Mobile social networking is currently a novel topic, conforming a challenging and exciting field of research which will undoubtedly receive much attention and investment from companies in the IT sector in years to come.

In this article we presented a novel application for mobile social networks, called BlueFriend, that seeks an alternative solution to support these networks by avoiding the use of the existing mobile telecommunications infrastructures. By relying on Bluetooth technology alone, users are able to participate in the social networks 24 hours a day without having to worry about signup and maintenance related costs, while assuring a high degree of privacy.

One of the main advantages of BlueFriend compared to other mobile social networks has to do with the interaction among users. Since the Bluetooth technology requires users to be close-by for communication to take place, BlueFriend avoids sedentarism by promoting that users travel and dwell in crowded areas to achieve a more active participation in the social network. Due to its context-aware behavior and it transparency to the user, not requiring any intervention at all, our approach embraces Weiser's vision [15] of an ubiquitous computing world.

By providing this application to our students and receiving their feedback we learned that introducing such pragmatic examples in classes can be quite effective at motivating and widening the horizon of future engineers towards novel technologies and concepts.

\section{Acknowledgments}

This work was partially supported by the Ministerio de Educación y Ciencia, Spain, under Grant TIN2008-06441C02-01.

\section{References}

[1] Facebook Inc., "Facebook." Retrieved March 2009, from http://www.facebook.com/.

[2] Twitter, Inc., “Twitter.” Retrieved March 2009, from http://twitter.com/.

[3] Jennifer Thom-Santelli, "Mobile Social Software: Facilitating Serendipity or Encouraging Homogeneity?,"
IEEE Pervasive Computing, vol. 6, no. 3, pp. 46-51, 2007.

[4] Nina D. Ziv and Bala Mulloth, "An Exploration on Mobile Social Networking: Dodgeball as a Case in Point," in Proceedings of the 6th International Conference on Mobile Business, (Toronto, Ontario, Canada), 2006.

[5] Chatschik Bisdikian, "An overview of the Bluetooth Wireless Technology," IEEE Communications Magazine, vol. 39, pp. 86-94, December 2001.

[6] P. Persson and Y. Jung, "Nokia sensor: from research to product," in Proceedings of the 2005 Conference on Designing For User Experience, (San Francisco, California, USA), 2005.

[7] Mobiluck, "Mobiluck Bluetooth Software." Retrieved November 2008, from http://www.mobiluck.com/.

[8] No Sleep Media LLC, "Brightkite.” Retrieved March 2009, from http://brightkite.com/.

[9] Loopt Inc., “Loopt.” Retrieved March 2009, from http://www.loopt.com/.

[10] E. Paulos and E. Goodman, "The familiar stranger: anxiety, comfort, and play in public places," in $\mathrm{CHI}$ '04: Proceedings of the 2004 conference on Human factors in computing systems, pp. 223-230, ACM Press, 2004.

[11] B. Davis and K. Karahalios, "Telelogs: a social communication space for urban environments," in MobileHCI '05: Proceedings of the 7th international conference on Human computer interaction with mobile devices \&amp; services, (New York, NY, USA), pp. 231-234, ACM, 2005.

[12] N. Eagle and A. Pentland, "Social serendipity: mobilizing social software," Pervasive Computing, IEEE, vol. 4, no. 2, pp. 28-34, 2005.

[13] R. Beale, "Supporting social interaction with smart phones," IEEE Pervasive Computing, vol. 4, pp. 3541, April 2005.

[14] D. Melinger, K. Bonna, M. Sharon, and M. SantRam, "Socialight: A Mobile Social Networking System," in Poster Proceedings of the 6th International Conference on Ubiquitous Computing (Ubicomp), (Nottingham, England), 2004.

[15] M. Weiser, "Some computer science issues in ubiquitous computing," Communications of the ACM, vol. 36, pp. 75-84, July 1993. 